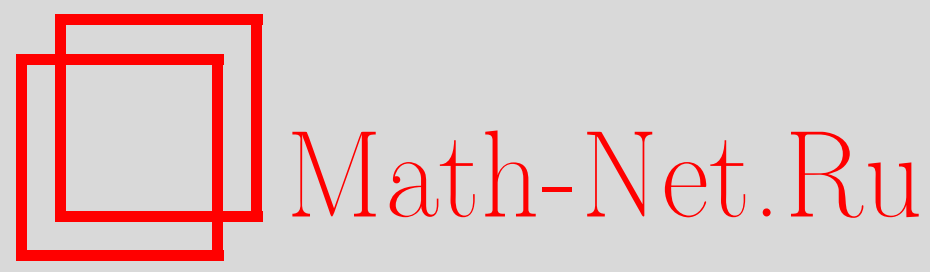

О. В. Сыромолотова, Произведения классов Фиттинга конечных групп, Матем. заметки, 2004, том 75, выпуск 2, 269-276

DOI: https://doi.org/10.4213/mzm33

Использование Общероссийского математического портала Math-Net.Ru подразумевает, что вы прочитали и согласны с пользовательским соглашением http://www.mathnet.ru/rus/agreement

Параметры загрузки:

IP: 34.227 .88 .159

26 апреля 2023 г., 10:59:47 


\title{
ПРОИЗВЕДЕНИЯ КЛАССОВ ФИТТИНГА КОНЕЧНЫХ ГРУПП
}

\author{
О.В. Сыромолотова
}

\begin{abstract}
В работе рассматриваются классы Фиттинга. В частности, показывается, что произведение $n$-кратно $\omega$-локальных классов $\Phi$ иттинга является $n$-кратно $\omega$-локальным классом Фиттинга.

Библиографория: 11 названий.
\end{abstract}

При конструировании новых классов групп одним из основных методов является факторизация классов групп. При этом возникает задача изучения полученных классов. Многие результаты этого направления установлены в теории формаций. В работах [1], [2] показывается, что если $\mathfrak{M}$ и $\mathfrak{H}$ - локальные формации, то формация $\mathfrak{M} \mathfrak{H}$ также является локальной. Аналогичньй результат получен для $\omega$-локальных формаций [3]. Кроме того, если ввести ограничение $\mathfrak{N}_{\pi(\mathfrak{M})} \subseteq \mathfrak{M}$, то произведение двух композиционных формаций является композиционной формацией [4]. Позже этот результат был распространен на $\Omega$-композиционные формации [5], [6]. В настоящей работе рассматриваются классы Фиттинга. В частности, показьвается, что произведение $n$-кратно $\omega$-локальных классов Фиттинга является $n$-кратно $\omega$-локальным классом Фиттинга, что дает ответ на вопрос 25 [3]. Отметим, что аналогичньй результат для $n$-кратно $\Omega$-канонических классов Фиттинга независимо получен Ведерниковым [7].

Рассматриваются только конечные группы. Необходимые определения и обозначения можно найти в [8]-[10]. В дальнейшем, $\omega$ обозначает некоторое непустое подмножество множества всех простых чисел $\mathbb{P}, \omega^{\prime}=\mathbb{P} \backslash \omega ; \Omega$-непустой подкласс класса всех конечных простых грушп $\mathfrak{I}, \Omega^{\prime}=\mathfrak{I} \backslash \Omega$. Функция

$$
f: \omega \cup\left\{\omega^{\prime}\right\} \rightarrow\{\text { классы Фиттинга групा }\}
$$

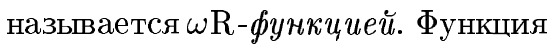

$$
\varphi: \mathbb{P} \rightarrow\{\text { непустые формации Фиттинга }\}
$$

назьвается PFR-функиией. Класс Фиттинга

$$
\mathfrak{F}=\omega \mathrm{R}(f, \varphi)=\left(G: O^{\omega}(G) \in f\left(\omega^{\prime}\right) \quad \text { и } G^{\varphi(p)} \in f(p) \quad \text { для всех } p \in \omega \cap \pi(G)\right)
$$

назьвается $\omega$-веерным, где $f$ и $\varphi$ - некоторые $\omega \mathrm{R}$-функция и $\mathbb{P F R}$-функция соответственно. При этом функция $f$ назьвается $\omega$-спутником, функция $\varphi-$ направлением 
$\omega$-веерного класса Фиттинга $\mathfrak{F}$. При рассмотрении различных направлений получаются различные классы Фиттинга. В частности, класс Фиттинга $\mathfrak{F}=\omega \mathrm{R}(f, \varphi)$ назьвается $\omega$-полным или, коротко, $\omega \mathrm{A}$-классом Фиттинга и обозначается $\mathfrak{F}=\omega \mathrm{AR}(f)$, если $\varphi(p)=\mathfrak{G}_{p^{\prime}}$ для любого $p \in \mathbb{P} ;$ класс Фиттинга $\mathfrak{F}=\omega \mathrm{R}(f, \varphi)$ называется $\omega$-локальным

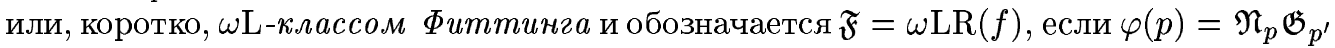
для любого $p \in \mathbb{P}$. Направление $\varphi \omega$-веерного класса Фиттинга назьвается $p n$-наnравлением, если $\varphi(p)=\varphi(p) \mathfrak{G}_{p^{\prime}}$ и $\varphi(p)$ не содержит простых неабелевых $p d$-групा для любого $p \in \mathbb{P}$. Функция

$$
f: \Omega \cup\left\{\Omega^{\prime}\right\} \rightarrow\{\text { классы Фиттинга групп }\},
$$

принимающая одинаковые значения на изоморфных группах из $\Omega$, назьвается $\Omega R-\phi y н к-$ uией. Класс Фиттинга $\mathfrak{F}$ называется $\Omega$-каноническим или, коротко, $\Omega \mathrm{K}$-классом Фиттинга и обозначается $\mathfrak{F}=\Omega \mathrm{KR}(f)$, если

$$
\mathfrak{F}=\left(G: O^{\Omega}(G) \in f\left(\Omega^{\prime}\right) \text { и } O^{\mathrm{A}, \mathrm{A}^{\prime}}(G) \in f(\mathrm{~A}) \quad \text { для всех } \mathrm{A} \in \Omega \cap K(G)\right) .
$$

При этом $\Omega \mathrm{R}$-функция $f$ называется $\Omega \mathrm{K}$-спутником класса Фиттинга $\mathfrak{F}$. Произведением классов Фиттинга $\mathfrak{M}$ и $\mathfrak{H}$ называется класс

$$
\mathfrak{M} \diamond \mathfrak{H}=\left\{G: G / G_{\mathfrak{M}} \in \mathfrak{H}\right\} .
$$

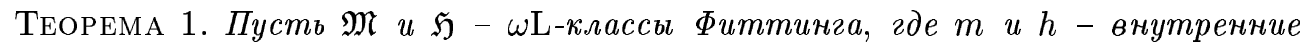
$\omega$-спутники классов $\mathfrak{M} и \mathfrak{H}$ соответственно. Тогда $\mathfrak{F}=\mathfrak{M} \diamond \mathfrak{H}-\omega \mathrm{L}-\kappa л а с с$ Фиттинга с $\omega$-спутником $f$ таким, что

$$
\begin{aligned}
& f\left(\omega^{\prime}\right)=\mathfrak{F} ; \quad f(p)=\mathfrak{M} \diamond h(p), \quad \text { ecлu } \quad p \in \omega \cap \pi(\mathfrak{H}) ; \\
& f(p)=m(p), \quad \text { если } \quad p \in \omega \backslash \pi(\mathfrak{H}) .
\end{aligned}
$$

ДокАЗАТЕЛЬСТВо. Пусть $\mathfrak{F}_{1}=\omega \operatorname{LR}(f)$, где $f-\omega$-спутник, описанный в формулировке леммы. Покажем, что $\mathfrak{F}=\mathfrak{F}_{1}$.

1) Докажем, что $\mathfrak{F} \subseteq \mathfrak{F}_{1}$. Пусть $G \in \mathfrak{F}$. Рассмотрим следующие случаи.

a) Предположим, что $\omega \cap \pi(G)=\varnothing$. Тогда $O^{\omega}(G)=G \in \mathfrak{F}=f\left(\omega^{\prime}\right)$, и из $p \in \omega \cap$ $\pi(G)=\varnothing$ следует, что $F^{p}(G) \in f(p)$. Таким образом, $G \in \mathfrak{F}_{1}$. В дальнейшем, будем считать, что $\omega \cap \pi(G) \neq \varnothing$.

b) Пусть $p \in \omega \cap \pi\left(G / G_{\mathfrak{M}}\right)$. Так как $\mathfrak{N}_{p} \mathfrak{G}_{p^{\prime}}$ является формацией и $\mathfrak{M}-$ класс Фиттинга, то в силу леммы 1.2 гл. 1 [9] и леммы 1.1 гл. 9 [8]

$$
\mathrm{F}^{p}(G) /\left(\mathrm{F}^{p}(G)\right)_{\mathfrak{M}}=F^{p}(G) / G_{\mathfrak{M}} \cap \mathrm{F}^{p}(G) \cong \mathrm{F}^{p}(G) G_{\mathfrak{M}} / G_{\mathfrak{M}}=\mathrm{F}^{p}\left(G / G_{\mathfrak{M}}\right) .
$$

Поскольку $G / G_{\mathfrak{M}} \in \mathfrak{H}$, то $F^{p}\left(G / G_{\mathfrak{M}}\right) \in h(p)$, и значит, $F^{p}(G) /\left(F^{p}(G)\right)_{\mathfrak{M}} \in h(p)$. Отсюда $F^{p}(G) \in \mathfrak{M} \diamond h(p)$. Так как $G / G_{\mathfrak{M}} \in \mathfrak{H}$, то $p \in \omega \cap \pi(\mathfrak{H})$, и, следовательно, $F^{p}(G) \in f(p)$.

c) Пусть $p \in[\omega \cap \pi(G)] \backslash \pi\left(G / G_{\mathfrak{M}}\right)$, т.е. $G / G_{\mathfrak{M}}$ является $p^{\prime}$-группой. Тогда по лемме $1[3] F^{p}(G)=F^{p}\left(G_{\mathfrak{M}}\right)$. Так как $G_{\mathfrak{M}} \in \mathfrak{M}$, то $F^{p}\left(G_{\mathfrak{M}}\right) \in m(p)$, и значит, $F^{p}(G) \in m(p)$; теперь

i) если $p \in \omega \cap \pi(\mathfrak{H})$, то

$$
F^{p}(G) \in m(p) \subseteq \mathfrak{M} \subseteq \mathfrak{M} \diamond h(p)=f(p) ;
$$

ii) если $p \in \omega \backslash \pi(\mathfrak{H})$, то

$$
F^{p}(G) \in m(p)=f(p) .
$$


Из b) и с) видим, что $F^{p}(G) \in f(p)$ для любого $p \in \omega \cap \pi(G)$. Кроме того, так как $G \in \mathfrak{F}$ и $O^{\omega}(G) \triangleleft G$, то $O^{\omega}(G) \in \mathfrak{F}=f\left(\omega^{\prime}\right)$. Отсюда $G \in \mathfrak{F}_{1}$. Следовательно, $\mathfrak{F} \subseteq \mathfrak{F}_{1}$.

2) Пусть $G \in \mathfrak{F}_{1} \backslash \mathfrak{F}$ и $G$ - групша минимального порядка с таким свойством. Тогда $G$ - комонолитическая группа с комонолитом $M=G_{\mathfrak{F}}$.

Из того, что $\mathfrak{M} \subseteq \mathfrak{F}$ и $G \notin \mathfrak{F}$, получаем $G \notin \mathfrak{M}$. Тогда $G_{\mathfrak{M}} \neq G$ и из комонолитичности групшы $G$ следует, что $G_{\mathfrak{M}} \subseteq M$. Кроме того, $G_{\mathfrak{M}}=M_{\mathfrak{M}}$, и поскольку $M \in \mathfrak{F}=\mathfrak{M} \diamond \mathfrak{H}$, то $M / M_{\mathfrak{M}}=M / G_{\mathfrak{M}} \in \mathfrak{H}$.

Допустим, $G / M$ не является $\omega$-группой. Тогда $G=O^{\omega}(G) \in f\left(\omega^{\prime}\right)=\mathfrak{F}$. Противоречие. Следовательно, $G / M$ является $\omega$-групой.

Если $G / M$ - неабелева группа и $p \in \pi(G / M)$, то, учитывая, что спутники $m$ и $h$ внутренние, получаем $G=F^{p}(G) \in f(p) \subseteq \mathfrak{M} \diamond \mathfrak{H}=\mathfrak{F}$. Противоречие.

Значит, $G / M$ является $p$-группой. Рассмотрим следующие возможные случаи.

a) Пусть $p \in \omega \cap \pi(\mathfrak{H})$. Тогда $F^{p}(G) \in f(p)=\mathfrak{M} \diamond h(p)$, т.е. $F^{p}(G) /\left(F^{p}(G)\right)_{\mathfrak{M}} \in$ $h(p)$. Ранее былопоказано, что $F^{p}(G) /\left(F^{p}(G)\right)_{\mathfrak{M}} \cong F^{p}\left(G / G_{\mathfrak{M}}\right)$. Отсюда $F^{p}\left(G / G_{\mathfrak{M}}\right) \in$ $h(p)$. Аналогично можно показать, что $F^{q}\left(G / G_{\mathfrak{M}}\right) \in h(q)$ для любого $q \in \omega \cap \pi(\mathfrak{H}) \cap$ $\pi(G)$. Поскольку $\pi\left(M / G_{\mathfrak{M}}\right) \subseteq \pi(\mathfrak{H})$ и $\pi(G / M) \subseteq \pi(\mathfrak{H})$, то $\pi\left(G / G_{\mathfrak{M}}\right) \subseteq \pi(\mathfrak{H})$, и значит, $F^{q}\left(G / G_{\mathfrak{M}}\right) \in h(q)$ для любого $q \in \omega \cap \pi\left(G / G_{\mathfrak{M}}\right)$. Кроме того, так как $\mathfrak{G}_{\omega}$ - формация, то по лемме 1.2 гл. 1 [9]

$$
O^{\omega}\left(G / G_{\mathfrak{M}}\right)=O^{\omega}(G) G_{\mathfrak{M}} / G_{\mathfrak{M}} \cong O^{\omega}(G) / O^{\omega}(G) \cap G_{\mathfrak{M}}=O^{\omega}(G) /\left(O^{\omega}(G)\right)_{\mathfrak{M}}
$$

Из $O^{\omega}(G) \triangleleft M \in \mathfrak{F}$, леммы $10[10]$ получаем

$$
O^{\omega}\left(G / G_{\mathfrak{M}}\right) \cong O^{\omega}(G) /\left(O^{\omega}(G)\right)_{\mathfrak{M}} \in \mathfrak{H}=h\left(\omega^{\prime}\right)
$$

Отсюда $G / G_{\mathfrak{M}} \in \mathfrak{H}$, и, следовательно, $G \in \mathfrak{M} \diamond \mathfrak{H}=\mathfrak{F}$. Противоречие.

b) Пусть $p \in \omega \backslash \pi(\mathfrak{H})$. Тогда $F^{p}(G)=O^{p}(G) \in m(p)$. Нетрудно показать, что $O^{p}(G) \subset G$. Поскольку $M / O^{p}(G) \triangleleft G / O^{p}(G) \in \mathfrak{N}_{p}$, то $M / O^{p}(G)$ является $q^{\prime}$-группой для любого $q \in \omega \cap \pi(G), q \neq p$. Тогда по лемме $1[3] F^{q}(M)=F^{q}\left(O^{p}(G)\right)$. Так как $O^{p}(G) \in m(p) \subseteq \mathfrak{M}$, то $F^{q}\left(O^{p}(G)\right) \in m(q)$ для любого $q \in \omega \cap \pi\left(O^{p}(G)\right), q \neq p$. Поскольку $M / O^{p}(G)-q^{\prime}$-групша, то $F^{q}\left(O^{p}(G)\right) \in m(q)$ для любого $q \in \omega \cap \pi(M)$, $q \neq p$. Аналогично, из того, что $G / M$ является $q^{\prime}$-группой, по лемме $1[3] F^{q}(G)=$ $F^{q}(M) \in m(q)$ для любого $q \in \omega \cap \pi(G), q \neq p$. Кроме того, поскольку $p \in \omega$, то $M / O^{p}(G) \in \mathfrak{N}_{p} \subseteq \mathfrak{G}_{\omega}$. Следовательно, $O^{\omega}(M)=O^{\omega}\left(O^{p}(G)\right)$, а так как $O^{p}(G) \in \mathfrak{M}$, то $O^{\omega}\left(O^{p}(G)\right) \in m\left(\omega^{\prime}\right)$. Из $G / M \in \mathfrak{G}_{\omega}$ следует, что $O^{\omega}(G)=O^{\omega}(M) \in m\left(\omega^{\prime}\right)$. Получили, что $G \in \omega \operatorname{LR}(m)=\mathfrak{M} \subseteq \mathfrak{M} \diamond \mathfrak{H}=\mathfrak{F}$. Противоречие. Значит, $\mathfrak{F}_{1} \subseteq \mathfrak{F}$.

Из 1) и 2) следует, что $\mathfrak{F}=\mathfrak{F}_{1}$. Теорема доказана.

ОПРЕДЕЛЕНИЕ 1 [3]. Класс Фиттинга $\mathfrak{F}$ назьвается $n$-кратно $\omega$-локальны $\mathcal{M}, n \geq 1$, если $\mathfrak{F}=\omega \operatorname{LR}(f)$, причем все непустые значения спутника $f$ являются $(n-1)$-кратно $\omega$-локальными классами Фиттинга. Произвольный класс Фиттинга считается 0-кратно $\omega$-локальным классом Фиттинга.

СлЕДСТВИЕ 1. Пусть $\mathfrak{M} u \mathfrak{H}-n$-кратно $\omega$-локальные классы Фиттинга. Тогда $\mathfrak{F}=\mathfrak{M} \diamond \mathfrak{H}$ является $n$-кратно $\omega$-локальным классом Фиттинга. 
ДокАЗАТЕЛЬСТВО проведем индукцией по $n$. При $n=0$ утверждение ввиду теоремы 1.12 гл. 9 [8] верно. При $n=1$ справедливость следствия 1 вытекает из леммы 10 [10] и теоремы 1 . Пусть утверждение следствия 1 выполняется для любых натуральных чисел, меньших $n$, и $\mathfrak{M}=\omega \operatorname{LR}(m), \mathfrak{H}=\omega \operatorname{LR}(h)$, причем все непустые значения спутников $m$ и $h$ суть $(n-1)$-кратно $\omega$-локальные классы Фиттинга. Ввиду леммы 10 [10] можем

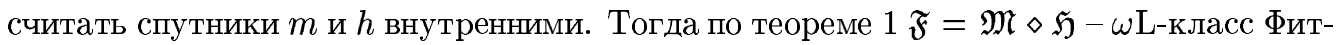
тинга с $\omega$-спутником $f$ таким, что $f\left(\omega^{\prime}\right)=\mathfrak{F} ; f(p)=\mathfrak{M} \diamond h(p)$, если $p \in \omega \cap \pi(\mathfrak{H})$; $f(p)=m(p)$, если $p \in \omega \backslash \pi(\mathfrak{H})$.

Так как $\mathfrak{M}$ и $\mathfrak{H}$ являются $(n-1)$-кратно $\omega$-локальными классами Фиттинга, то по предположению индукции $\mathfrak{F}$ является $(n-1)$-кратно $\omega$-локальным классом Фиттинга. Также по индукции заключаем, что $\mathfrak{M} \diamond h(p)$ является $(n-1)$-кратно $\omega$-локальным классом Фиттинга. Таким образом, все непустые значения спутника $f$ являются $(n-1)$-кратно $\omega$-локальными классами Фиттинга. Тогда по определению $1 \mathfrak{F}$ является $n$-кратно $\omega$-локальным классом Фиттинга. Следствие доказано.

ТЕОРема 2. Пусть $\mathfrak{F}=\mathfrak{M} \diamond \mathfrak{H}, \mathfrak{H}=\omega \mathrm{R}(h, \varphi)$ с внутренним $\omega$-спутником $h$ и рп-направлением $\varphi, \mathfrak{M}$ - непустой класс Фиттинга, причем $m$ - минимальный $\omega$-спутник класса Фиттинга $\omega \mathrm{R}(\mathfrak{M}, \varphi)$. Если F является $\omega$-веерным классом Фиттинга с рп-направлением $\varphi$, то $\mathfrak{F}$ обладает $\omega$-спутником $f$ таким, что

$$
\begin{aligned}
& f\left(\omega^{\prime}\right)=\mathfrak{F}, \quad f(p)=\mathfrak{M} \diamond h(p), \quad \text { ecлu } \quad p \in \omega \cap \pi(\mathfrak{H}), \\
& f(p)=m(p), \quad \text { ecлu } \quad p \in \omega \backslash \pi(\mathfrak{H}) .
\end{aligned}
$$

ДокАЗАТЕЛЬСТво. Пусть $\mathfrak{F}-\omega$-веерньй класс Фиттинга с $p n$-направлением $\varphi, \mathfrak{F}_{1}=$ $\omega \mathrm{R}(f, \varphi)$, где $f-\omega$-спутник, описанньй в формулировке теоремы. Покажем, что $\mathfrak{F}=\mathfrak{F}_{1}$.

1) Докажем, что $\mathfrak{F} \subseteq \mathfrak{F}_{1}$. Пусть $G \in \mathfrak{F}$. Рассмотрим следуюшие случаи.

а) Предположим, что $\omega \cap \pi(G)=\varnothing$. Так как $G \in \mathfrak{F}$, то $G=O^{\omega}(G) \in \mathfrak{F}=f\left(\omega^{\prime}\right)$. Кроме того, из $p \in \omega \cap \pi(G)=\varnothing$ следует, что $G^{\varphi(p)} \in f(p)$. Таким образом, $G \in \mathfrak{F}_{1}$. В дальнейшем, будем считать, что $\omega \cap \pi(G) \neq \varnothing$.

b) Пусть $p \in \omega \cap \pi\left(G / G_{\mathfrak{M}}\right)$. Так как $\varphi(p)$ является формацией и $\mathfrak{M}-$ классом Фиттинга, то в силу леммы 1.2 гл. 1 [9] и леммы 1.1 гл. 9 [8]

$$
G^{\varphi(p)} /\left(G^{\varphi(p)}\right)_{\mathfrak{M}}=G^{\varphi(p)} / G_{\mathfrak{M}} \cap G^{\varphi(p)} \cong G^{\varphi(p)} G_{\mathfrak{M}} / G_{\mathfrak{M}}=\left(G / G_{\mathfrak{M}}\right)^{\varphi(p)} .
$$

Поскольку $G / G_{\mathfrak{M}} \in \mathfrak{H}$, то $\left(G / G_{\mathfrak{M}}\right)^{\varphi(p)} \in h(p)$, и значит, $G^{\varphi(p)} /\left(G^{\varphi(p)}\right)_{\mathfrak{M}} \in h(p)$. Отсюда $G^{\varphi(p)} \in \mathfrak{M} \diamond h(p)$. Так как $G / G_{\mathfrak{M}} \in \mathfrak{H}$, то $p \in \pi(\mathfrak{H}) \cap \omega$, и следовательно, $G^{\varphi(p)} \in f(p)$.

c) Пусть $p \in[\omega \cap \pi(G)] \backslash \pi\left(G / G_{\mathfrak{M}}\right)$, т.е. $G / G_{\mathfrak{M}}$ является $p^{\prime}$-грушпой. Тогда по лемме $11[7] G^{\varphi(p)}=\left(G_{\mathfrak{M}}\right)^{\varphi(p)}$. Так как $G_{\mathfrak{M}} \in \mathfrak{M} \subseteq \omega \mathrm{R}(\mathfrak{M}, \varphi)$, то $\left(G_{\mathfrak{M}}\right)^{\varphi(p)} \in m(p)$, и значит, $G^{\varphi(p)} \in m(p)$; далее,

i) если $p \in \omega \cap \pi(\mathfrak{H})$, то

$$
G^{\varphi(p)}=\left(G_{\mathfrak{M}}\right)^{\varphi(p)} \triangleleft G_{\mathfrak{M}} \in \mathfrak{M} \subseteq \mathfrak{M} \diamond h(p)=f(p) ;
$$

ii) если $p \in \omega \backslash \pi(\mathfrak{H})$, то

$$
G^{\varphi(p)} \in m(p)=f(p) .
$$


Из b) и с) следует, что $G^{\varphi(p)} \in f(p)$ для любого $p \in \omega \cap \pi(G)$. Кроме того, так как $G \in \mathfrak{F}$, то $O^{\omega}(G) \in \mathfrak{F}=f\left(\omega^{\prime}\right)$. Отсюда $G \in \mathfrak{F}_{1}$. Следовательно, $\mathfrak{F} \subseteq \mathfrak{F}_{1}$.

2) Пусть $G \in \mathfrak{F}_{1} \backslash \mathfrak{F}$ и $G$ - группа минимального порядка с таким свойством. Тогда $G$ - комонолитическая группа с комонолитом $M=G_{\mathfrak{F}}$. Проводя рассуждения, аналогичные рассуждениям в теореме 1 , нетрудно проверить, что $M / G_{\mathfrak{M}} \in \mathfrak{H}$ и $G / M$ является $\omega$-группой.

Если $G / M$ - неабелева группа и $p \in \pi(G / M)$, то, поскольку $\varphi$ является $p n$-направлением, получаем $G=G^{\varphi(p)}$. Из того, что $m$ и $h$ являются внутренними спутниками и $\omega \mathrm{R}(\mathfrak{M}, \varphi) \subseteq \mathfrak{F}$, следует, что $G=G^{\varphi(p)} \in f(p) \subseteq \mathfrak{F}$. Противоречие.

Значит, $G / M$ является простой $p$-группой. Рассмотрим следуюшие возможные случаи.

a) Пусть $p \in \omega \cap \pi(\mathfrak{H})$. Тогда $G^{\varphi(p)} \in f(p)=\mathfrak{M} \diamond h(p)$, т.е.

$$
G^{\varphi(p)} /\left(G^{\varphi(p)}\right)_{\mathfrak{M}} \in h(p) .
$$

Ранее было показано, что $G^{\varphi(p)} /\left(G^{\varphi(p)}\right)_{\mathfrak{M}} \cong\left(G / G_{\mathfrak{M}}\right)^{\varphi(p)}$, т.е. $\left(G / G_{\mathfrak{M}}\right)^{\varphi(p)} \in h(p)$. Аналогично можно показать, что $\left(G / G_{\mathfrak{M}}\right)^{\varphi(q)} \in h(q)$ для любого $q \in \omega \cap \pi(\mathfrak{H}) \cap \pi(G)$. Поскольку $\pi\left(G / G_{\mathfrak{M}}\right) \subseteq \pi(\mathfrak{H})$, то $\left(G / G_{\mathfrak{M}}\right)^{\varphi(q)} \in h(q)$ для любого $q \in \omega \cap \pi\left(G / G_{\mathfrak{M}}\right)$. Как и в теореме $1 O^{\omega}\left(G / G_{\mathfrak{M}}\right) \in h\left(\omega^{\prime}\right)$. Значит, $G / G_{\mathfrak{M}} \in \mathfrak{H}$, и, следовательно, $G \in \mathfrak{M} \diamond$ $\mathfrak{H}=\mathfrak{F}$. Противоречие.

b) Пусть $p \in \omega \backslash \pi(\mathfrak{H})$. Тогда $G^{\varphi(p)} \in f(p)=m(p)$. Пусть $f_{1}-$ минимальньй $\omega$-спутник класса Фиттинга $\mathfrak{F}$. Так как $\mathfrak{M} \subseteq \mathfrak{F}$, то $\omega \mathrm{R}(\mathfrak{M}, \varphi) \subseteq \mathfrak{F}$. Тогда по следствию 12.1 [10] $m \leq f_{1}$, и значит, $G^{\varphi(p)} \in m(p) \subseteq f_{1}(p)$. Так как $G / M$ является $\omega$-группой, то $O^{\omega}(G)=$ $O^{\omega}(M)$. Поскольку $M \in \mathfrak{F}$, то

$$
O^{\omega}(G)=O^{\omega}(M) \in f_{1}\left(\omega^{\prime}\right)
$$

Пусть $q \in \omega \cap \pi(G), q \neq p$. Так как $G / M-q^{\prime}$-группа, то $G^{\varphi(q)}=M^{\varphi(q)}$. Из $M \in \mathfrak{F}$ следует, что $G^{\varphi(q)}=M^{\varphi(q)} \in f_{1}(q)$ для любого $q \in \omega \cap \pi(M), q \neq p$, и значит, любого $q \in \omega \cap \pi(G), q \neq p$. Отсюда $G \in \mathfrak{F}$. Противоречие. Значит, $\mathfrak{F}_{1} \subseteq \mathfrak{F}$.

Из 1) и 2) следует, что $\mathfrak{F}=\mathfrak{F}_{1}$. Теорема доказана.

СлЕДСТВИЕ 2. Пусть $\mathfrak{F}=\mathfrak{M} \diamond \mathfrak{H}, \mathfrak{H}=\omega \mathrm{AR}(h)$ с внутренним $\omega$-спутником $h, \mathfrak{M}$ - непустой класс Фиттинга, причем $m$ - минимальный $\omega$-спутник класса Фиттинга $\omega \mathrm{AR}(\mathfrak{M})$. Если $\mathfrak{F}$ является $\omega$-полным классом Фиттинга, то $\mathfrak{F}$ обладает $\omega$-спутником $f$ таким, что

$$
\begin{aligned}
& f\left(\omega^{\prime}\right)=\mathfrak{F}, \quad f(p)=\mathfrak{M} \diamond h(p), \quad \text { ecлu } \quad p \in \omega \cap \pi(\mathfrak{H}), \\
& f(p)=m(p), \quad \text { ecлu } \quad p \in \omega \backslash \pi(\mathfrak{H}) .
\end{aligned}
$$

СлЕДСТВИЕ 3. Пусть $\mathfrak{F}=\mathfrak{M} \diamond \mathfrak{H}, \mathfrak{H}=\omega \operatorname{LR}(h)$ с внутренним $\omega$-спутником $h, \mathfrak{M}$ - непустой класс Фиттинга, причем $m$ - минимальный $\omega$-спутник класса Фиттинга $\omega \mathrm{LR}(\mathfrak{M})$. Если $\mathfrak{F}$ является $\omega$-локальным классом Фиттинга, то Ғ обладает $\omega$-спутником $f$ таким, что

$$
\begin{aligned}
& f\left(\omega^{\prime}\right)=\mathfrak{F}, \quad f(p)=\mathfrak{M} \diamond h(p), \quad \text { eслu } \quad p \in \omega \cap \pi(\mathfrak{H}), \\
& f(p)=m(p), \quad \text { ecлu } \quad p \in \omega \backslash \pi(\mathfrak{H}) .
\end{aligned}
$$


Теорема 3. Пусть $\mathfrak{M} u \mathfrak{H}-\Omega \mathrm{K}$-классы Фиттинга, где $m u h$ - внутренние $\Omega$-спутники классов $\mathfrak{M}$ и $\mathfrak{H}$ соответственно. Тогда $\mathfrak{F}=\mathfrak{M} \diamond \mathfrak{H}-\Omega \mathrm{K}$-класс Фиттинга с $\Omega$-спутником $f$ таким, что

$$
\begin{aligned}
& f\left(\Omega^{\prime}\right)=\mathfrak{F}, \quad f(A)=\mathfrak{M} \diamond h(A), \quad \text { если } \quad A \in \Omega \cap K(\mathfrak{H}), \\
& f(A)=m(A), \quad \text { если } \quad A \in \Omega \backslash K(\mathfrak{H}) .
\end{aligned}
$$

ДокАЗАТЕЛЬСТво. Пусть $\mathfrak{F}_{1}=\Omega \mathrm{KR}(f)$, где $f-\Omega \mathrm{K}$-спутник, описанньй в формулировке теоремы. Покажем, что $\mathfrak{F}=\mathfrak{F}_{1}$.

1) Докажем, что $\mathfrak{F} \subseteq \mathfrak{F}_{1}$. Пусть $G \in \mathfrak{F}$. Рассмотрим следуюшие случаи.

a) Если $\Omega \cap K(G)=\varnothing$, то $O^{\Omega}(G)=G \in \mathfrak{F}=f\left(\Omega^{\prime}\right)$, из $A \in \Omega \cap K(G)=\varnothing$ следует, что $O^{A, A^{\prime}}(G) \in f(A)$, и значит, $G \in \mathfrak{F}_{1}$. В дальнейшем полагаем, что $\Omega \cap K(G) \neq \varnothing$.

b) Пусть $A \in \Omega \cap K\left(G / G_{\mathfrak{M}}\right)$. Так как $\mathfrak{G}_{A} \mathfrak{G}_{A^{\prime}}$ является формацией и $\mathfrak{M}$ - классом Фиттинга, то в силу леммы 1.2 гл. 1 [9] и леммы 1.1 гл. 9 [8]

$$
O^{A, A^{\prime}}(G) /\left(O^{A, A^{\prime}}(G)\right)_{\mathfrak{M}} \cong O^{A, A^{\prime}}\left(G / G_{\mathfrak{M}}\right) .
$$

Поскольку $G / G_{\mathfrak{M}} \in \mathfrak{H}$, то $O^{A, A^{\prime}}\left(G / G_{\mathfrak{M}}\right) \in h(A)$, и значит, $O^{A, A^{\prime}}(G) /\left(O^{A, A^{\prime}}(G)\right)_{\mathfrak{M}} \in$ $h(A)$, т.е.

$$
O^{A, A^{\prime}}(G) \in \mathfrak{M} \diamond h(A)=f(A) .
$$

c) Пусть

$$
A \in[\Omega \cap K(G)] \backslash K\left(G / G_{\mathfrak{M}}\right) .
$$

Тогда $G / G_{\mathfrak{M}}$ является $A^{\prime}$-группой и, значит, по лемме 11 [7] $O^{A, A^{\prime}}(G)=O^{A, A^{\prime}}\left(G_{\mathfrak{M}}\right)$. Так как $G_{\mathfrak{M}} \in \mathfrak{M}$, то $O^{A, A^{\prime}}\left(G_{\mathfrak{M}}\right) \in m(A)$, и следовательно, $O^{A, A^{\prime}}(G) \in m(A)$. Нетрудно проверить, что в этом случае $O^{A, A^{\prime}}(G) \in f(A)$.

Из b) и с) видим, что

$$
O^{A, A^{\prime}}(G) \in f(A) \quad \text { для любого } A \in \Omega \cap K(G) .
$$

Кроме того, так как $G \in \mathfrak{F}$, то $O^{\Omega}(G) \in \mathfrak{F}=f\left(\Omega^{\prime}\right)$. Отсюда $G \in \mathfrak{F}_{1}$. Следовательно, $\mathfrak{F} \subseteq \mathfrak{F}_{1}$.

2) Пусть $G \in \mathfrak{F}_{1} \backslash \mathfrak{F}$ и $G$ - группа минимального порядка с таким свойством. Тогда $G$ - комонолитическая группа с комонолитом $M=G_{\mathfrak{F}}$, причем можно показать, что $M / G_{\mathfrak{M}} \in \mathfrak{H}$ и $G / M$ является $\Omega$-группой. Пусть $G / M \cong A$. Рассмотрим следуюшие возможные случаи.

a) Пусть $A \in \Omega \cap K(\mathfrak{H})$. Тогда $O^{A, A^{\prime}}(G) \in f(A)=\mathfrak{M} \diamond h(A)$, т.е.

$$
O^{A, A^{\prime}}(G) /\left(O^{A, A^{\prime}}(G)\right)_{\mathfrak{M}} \in h(A) .
$$

Так как

$$
O^{A, A^{\prime}}(G) /\left(O^{A, A^{\prime}}(G)\right)_{\mathfrak{M}} \cong O^{A, A^{\prime}}\left(G / G_{\mathfrak{M}}\right),
$$

то $O^{A, A^{\prime}}\left(G / G_{\mathfrak{M}}\right) \in h(A)$. Аналогично можно показать, что

$$
O^{B, B^{\prime}}\left(G / G_{\mathfrak{M}}\right) \in h(B) \quad \text { для любой } B \in \Omega \cap K(\mathfrak{H}) \cap K(G) .
$$


Поскольку $K\left(M / G_{\mathfrak{M}}\right) \subseteq K(\mathfrak{H})$ и $A \in K(\mathfrak{H})$, то $K\left(G / G_{\mathfrak{M}}\right) \subseteq K(\mathfrak{H})$, и значит,

$$
O^{B, B^{\prime}}\left(G / G_{\mathfrak{M}}\right) \in h(B) \text { для любой } B \in \Omega \cap K\left(G / G_{\mathfrak{M}}\right) \text {. }
$$

Кроме того, так как $\mathfrak{G}_{\Omega}-$ формация, то в силу леммы 1.2 гл. 1 [9]

$$
O^{\Omega}\left(G / G_{\mathfrak{M}}\right) \cong O^{\Omega}(G) /\left(O^{\Omega}(G)\right)_{\mathfrak{M}}
$$

Поскольку $O^{\Omega}(G) \triangleleft M \in \mathfrak{F}$, то $O^{\Omega}(G) /\left(O^{\Omega}(G)\right)_{\mathfrak{M}} \in \mathfrak{H}$, и значит, ввиду леммы 11 [11]

$$
O^{\Omega}\left(G / G_{\mathfrak{M}}\right) \in \mathfrak{H}=h\left(\Omega^{\prime}\right) .
$$

Отсюда $G / G_{\mathfrak{M}} \in \mathfrak{H}$, и, следовательно, $G \in \mathfrak{M} \diamond \mathfrak{H}=\mathfrak{F}$. Противоречие.

b) Пусть $A \in \Omega \backslash K(\mathfrak{H})$. Тогда

$$
O^{A, A^{\prime}}(G)=O^{A}(G) \in f(A)=m(A) .
$$

Нетрудно показать, что $O^{A}(G) \subset G$. Поскольку $M / O^{A}(G) \triangleleft G / O^{A}(G) \in \mathfrak{G}_{A}$, то $M / O^{A}(G)$ является $B^{\prime}$-группой для любой $B \in \Omega \cap K(G), B \not A$. Тогда

$$
O^{B, B^{\prime}}(M)=O^{B, B^{\prime}}\left(O^{A}(G)\right) .
$$

Так как $O^{A}(G) \in m(A) \subseteq \mathfrak{M}$, то $O^{B, B^{\prime}}\left(O^{A}(G)\right) \in m(B)$ для любой $B \in \Omega \cap K\left(O^{A}(G)\right)$, $B \not A$. Поскольку $M / O^{A}(G)-B^{\prime}$-группа, то

$$
O^{B, B^{\prime}}\left(O^{A}(G)\right) \in m(B) \quad \text { для любой } B \in \Omega \cap K(M), \quad B \not A .
$$

Аналогично из того, что $G / M$ является $B^{\prime}$-группой, получаем, что

$$
O^{B, B^{\prime}}(G)=O^{B, B^{\prime}}(M) \in m(B) \quad \text { для любой } \quad B \in \Omega \cap K(G), \quad B \not A \text {. }
$$

Поскольку $A \in \Omega$ и $O^{A}(G) \in \mathfrak{M}$, то ввиду леммы 7 [11] получаем

$$
O^{\Omega}(G)=O^{\Omega}(M)=O^{\Omega}\left(O^{A}(G)\right) \in m\left(\Omega^{\prime}\right) .
$$

Следовательно,

$$
G \in \Omega \mathrm{KR}(m)=\mathfrak{M} \subseteq \mathfrak{M} \diamond \mathfrak{H}=\mathfrak{F} .
$$

Противоречие. Значит, $\mathfrak{F}_{1} \subseteq \mathfrak{F}$.

Из 1) и 2) следует, что $\mathfrak{F}=\mathfrak{F}_{1}$. Теорема доказана.

ОПРЕДЕЛЕниЕ 2 . Следуя [3], класс Фиттинга $\mathfrak{F}$ назовем $n$-кратно $\Omega$-каноническим, $n \geq 1$, если $\mathfrak{F}=\Omega \mathrm{KR}(f)$, причем все непустые значения спутника $f$ являются $(n-1)$-кратно $\Omega$-каноническими классами Фиттинга. Произвольньй класс Фиттинга будем считать 0 -кратно $\Omega$-каноническим классом Фиттинга.

СлЕДСТвИЕ 4. Пусть $\mathfrak{M}$ и $\mathfrak{H}-n$-кратно $\Omega$-канонические классы Фиттинга. Тогда $\mathfrak{F}=\mathfrak{M} \diamond \mathfrak{H}$ является $n$-кратно $\Omega$-каноническим классом Фиттинга. 
ДокАЗАТЕЛЬСТВО проведем индукцией по $n$. При $n=0$ утверждение ввиду теоремы 1.12 гл. 9 [8] верно. При $n=1$ справедливость следствия 4 вытекает из леммы 11 [11] теоремы 3. Пусть утверждение следствия 4 вьполняется для любых натуральных чисел, меньших $n$, и $\mathfrak{M}=\Omega \mathrm{KR}(m), \mathfrak{H}=\Omega \mathrm{KR}(h)$, причем все непустые значения спутников $m$ и $h$ суть $(n-1)$-кратно $\Omega$-канонические классы Фиттинга. Ввиду леммы 11 [11] можем считать спутники $m$ и $h$ внутренними. Тогда по теореме $3 \mathfrak{F}=\mathfrak{M} \diamond \mathfrak{H}-\Omega$ К-класс Фиттинга с $\Omega$-спутником $f$ таким, что

$$
\begin{aligned}
f\left(\Omega^{\prime}\right)=\mathfrak{F}, \quad f(A) & =\mathfrak{M} \diamond h(A), \\
f(A) & =m(A), \quad \text { если } \quad A \in \Omega \cap K(\mathfrak{H}), \\
& \text { если } \quad A \in \Omega \backslash K(\mathfrak{H}) .
\end{aligned}
$$

Так как $\mathfrak{M}$ и $\mathfrak{H}$ являются $(n-1)$-кратно $\Omega$-каноническими классами Фиттинга, то по предположению индукции $\mathfrak{F}$ является $(n-1)$-кратно $\Omega$-каноническим классом Фиттинга. Также по индукции заключаем, что $\mathfrak{M} \diamond h(p)$ является $(n-1)$-кратно $\Omega$-каноническим классом Фиттинга. Таким образом, все непустые значения спутника $f$ являются $(n-1)$-кратно $\Omega$-каноническими классами Фиттинга. Тогда по определению $2 \mathfrak{F}$ является $n$-кратно $\Omega$-каноническим классом Фиттинга. Следствие доказано.

\section{СПИСОК ЦИТИРОВАННОЙ ЛИТЕРАТУРЫ}

[1] Шеметков Л.А. О произведении формаций // Докл. АН БССР. 1984. Т. 28. № 2. C. $101-103$.

[2] Шеметков Л.А. Экраны произведения формаций // Докл. АН БССР. 1981. Т. 25. № 8 . C. $677-680$.

[3] Скиба А. Н., Шеметков Л. А. Кратно $\omega$-локальные формации и классы Фиттинга конечных групп. Препринт № 63. Гомель: ГГУ, 1997.

[4] Скиба А.Н., Шеметков Л. А. Формации алгебраических систем. М.: Наука, 1989.

[5] Еловиков А. Б. Кратно $\Omega$-композиционные формации групп. Препринт № 3. Брянск: БГПУ, 1999.

[6] Скиба А.Н., Шеметков Л.А.Кратно L-композиционные формации конечных групп // Укр. матем. ж. 2000. Т. 52. №6. С. 783-797.

[7] Ведерников В.А. Максимальные спутники $\Omega$-расслоенных формаций и классов Фиттинга. Препринт № 1. Москва: МГПУ, 2001.

[8] Doerk K., Hawkes T. Finite Soluble Groups. Berlin-New York: Walter de Gruyter, 1992.

[9] Шеметков Л. А. Формации конечных групп. М.: Наука, 1978.

[10] Ведерников В.А., Сорокина М. М. $\omega$-веерные формации и классы Фиттинга конечных групп. Препринт № 6. Брянск: БГПУ, 1999.

[11] Ведерников В.А., Сорокина М. М. $\Omega$-расслоенные формации и классы Фиттинга конечных групп. Препринт № 5. Брянск: БГПУ, 1999. 